\title{
Intelligent Controller Design for a Sustainable Energy System
}

\author{
Kary Thanapalan, Ewen Constant \\ Faculty of Computing, Engineering and Sciences, University of South Wales, Pontypridd CF37 1DL, UK \\ Phone/Fax number: +0044 1443 482545, E-mail: kary.thanapalan@southwales.ac.uk
}

\begin{abstract}
This paper discusses an intelligent control system design for a sustainable energy system. This work also described an optimal design and implementation of an efficient selfsustainable energy system with consideration of energy storage options for excess energy produced by renewable energy sources.
\end{abstract}

\section{Key words}

Intelligent control, sustainable energy, power management, energy system.

\section{Introduction}

In recent years, the importance of the development of new self-sustainable energy system has been recognised globally, due to the rapid growth in global energy demand coupled with limited supply of fossil fuels [1].

Housing and buildings in the countryside consume a significant amount of primary energy for thermal or electrical power [2]. This sector is very important to Wales's economy and its sustainability due to its geographical place in the world. Wales is home to many historical sites, countryside buildings and houses, which are spread throughout the countryside [3]. Powering these sites may prove to be problematic, as power cables will disturb the landscape and prove costly. Using a solar powered self-sustainable energy system may resolve these problems.

Sustainable energy system can be either stand-alone or grid connected, or a hybridisation of both. Over the past decade, the development and application of sustainable energy system has increased significantly [3]. A significant amount of research work has addressed various topics for sustainable energy system; and includes modelling and simulation to analyse the complex interactions within the system and with exogenous factors. In addition, the application of control, in trying to maintain and evaluate optimal system performance has been of interest [3 -6].
The key issue with using renewable energies to supply the grid, is the fluctuational production; due to dependency on environmental factors such as wind and solar radiation [7]. Nevertheless, the supply side cannot react flexibly to the demand; which may result in an overproduction and supply to the grid [8]. Decoupling the production from the demand side maybe a solution; by storing the overproduced energy and then utilising it when required during peak time or when an insufficient production of energies occurs. Therefore, it is important to have a suitable energy storage system.

\section{Energy Storage Systems}

There are three distinct options of energy storage solutions to be considered and researched, whilst developing a sustainable grid connected energy system. In order to maximise the use of renewable energies detailed analysis must be carried out to maximise opportunities in the capture, storage and management of energy.

\section{a) Supercapacitor}

Supercapacitors, work on a principle that is, storing energy in an electric field that is created when charges of opposites sign are held separated from each other [9]. Supercapacitors have the ability to withstand a high volume of charge and discharge cycles without losing energy storage capability. However, the main problem of supercapacitors is their low energy density, therefore the storage capacity is limited, i.e., the amount of energy supercapacitors can store per unit weight is low compared to batteries. Nevertheless, life expectancy of supercapacitors is longer than the batteries, but the cost is still restrictively high [10]. Due to its high rate charge and discharge capability, it is useful to provide short duration power requirements [9].

\section{b) Batteries}

In relation to energy storage for stand-alone and selfsustainable renewable energy systems, Lead acid batteries are still ahead of the game in terms of energy storage / 
cost comparison, Lithium Ion batteries are continues to reduce in price, and now the market is approaching a position where Lithium-Ion batteries are able to compete on price [3].

Battery energy storage system are mainly used for household or smaller industrial applications, to store excess renewable energy and good for self-sustainable renewable energy systems, since they can be used independently as possible from the grid [11]. This is because there are only small periods, where it has to act as a buffer and the peak demand is low, compared to the grid. However, on the long run energy lost in batteries are unavoidable. Moreover, large batteries are heavy, take up a lot of space, and therefore not useful for many large scale applications. Nevertheless, in order to cope with the larger scale storage issues, industry is beginning to develop large battery packs. For example, Tesla in Australia, built a $1000 \mathrm{MW}$ Li-Ion battery pack with a storage capacity of $120 \mathrm{MWh}$ and it is connected to a nearby wind farm (Fig.1) which is operated by Neoen [12]. The key issue with the use of batteries in large-scale systems is the finite availability of the (rare) materials.

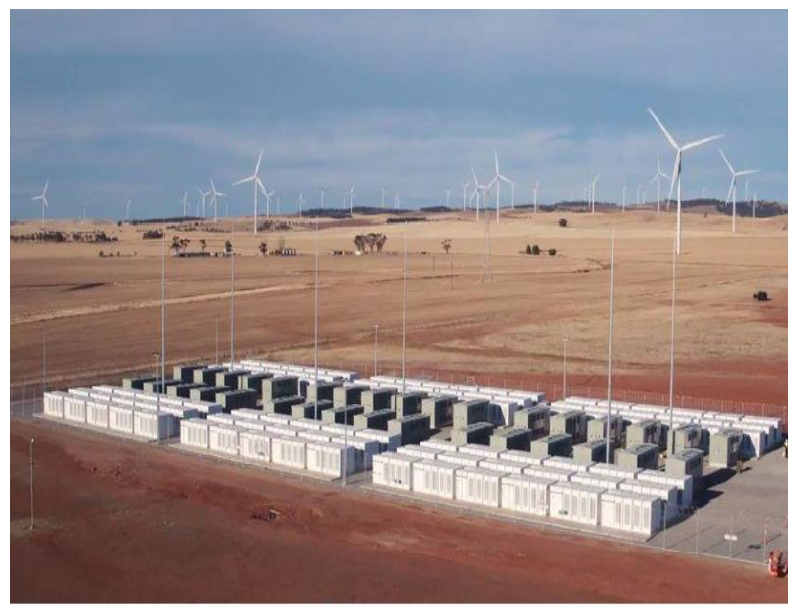

Fig.1. Large-scale battery storage system [12]

\section{c) Power to Gas Methods.}

Concerning the large-scale storage management, it is important to discuss the development of a new system, tailored for large-scale storage [13]. P2G energy storage method is a process of converting excess renewable energy supply into (hydrogen) gas by rapid response electrolysis and its subsequent injection into the gas distribution network [13].

The P2G system is a connection of multiple technologies; it includes feed-in, storage and supply technologies for different usage [14]. Storage of the energy carrier in gaseous state is the key part of P2G method. The P2G method can use the existing infrastructure and may be able to substituting conventional fuel or natural gas in the mobility and other sectors [13].
In P2G applications, hydrogen and methane are the producible gases [11]. Methanation is one process step behind the hydrogen, which reduces its effectiveness; however, it increases the usability for different sectors [14]. The more process steps involved, the more losses will appear during the chain of applications. This may be the key issue to be resolve for the future; to increase the efficiency and reduce the costs - particularly for methanation.

From the above analysis, it is clear that the battery energy storage system, is the best energy storage option for the domestic self-sustainable energy system described in this paper.

\section{System configuration}

The common self-sustainable energy system architecture will have the following subsystems, which are, renewable energy source (PV modules), energy storage subsystem (battery pack), power management system (controllers) and tasks (load). For illustration purpose a structural block diagram for a proposed flexible solar energy system is shown in Fig.2. The solid black arrows represent the electricity flow; the dotted grey arrow represents the control signals.

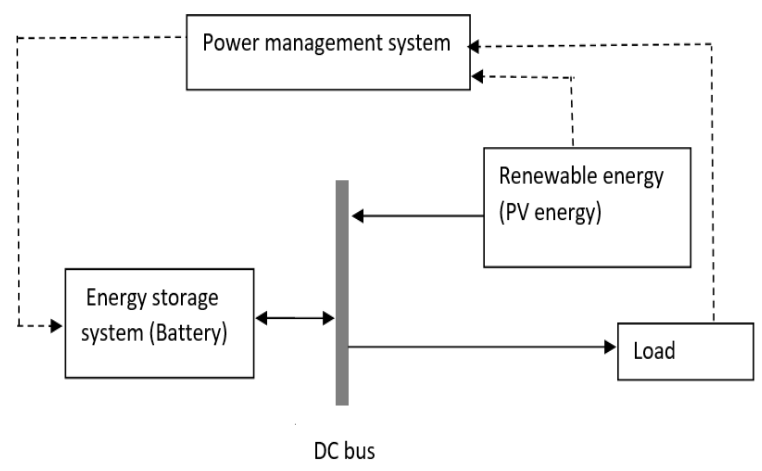

Fig.2. System configuration of the solar powered selfsustainable energy system.

In this study, a two-storey countryside house in Wales is assumed to be as a reference load profile. A photovoltaic array is considered as the main power source to a system which enables it to meet a predefined load demand. Due to the inherent intermittency of solar irradiation, a battery pack is included, but not only to smooth the fluctuation of the solar power generated, when such smoothing is necessary, it is also act as a primary energy storage when excess power is available. Furthermore, when there is a shortage of direct solar power, the battery pack will feed back the stored energy into the system. 


\section{A. System model}

Several researchers have addressed the development of an accurate system model, and the design and application of such systems [6, 15]. In this paper, a common but accurate PV model presented in [15] is adopted. The model of the PV system is based on an equivalent circuit, which consists of a current source, a diode and a series resister as shown in Figure 3.

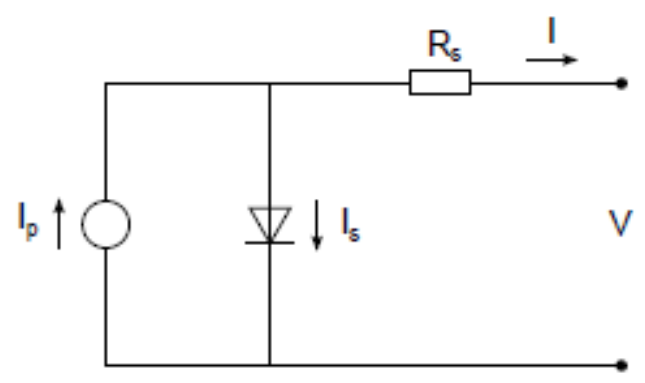

Fig.3. Equivalent circuit for PV array

The typical current-voltage (I-V) characteristic for PV cell is expressed in equation (1)

$$
I=I_{p}-I_{s}\left(\exp \left(V+I R_{s} / \varepsilon V_{t}\right)-1\right)
$$

where $I_{p}$ is the photo current, $I_{s}$ is the reverse saturation current which is affected by the temperature of the PV cell, $V$ is the cell voltage, $\varepsilon$ is the ideality factor which is approximately equal to $1, V_{t}$ is the thermal voltage and its given by;

$$
V_{t}=k_{B} T / q
$$

With the Boltzmann constant $k_{B}=1.38 \times 10^{-23} J^{-1}$; $T$ is the absolute temperature of the diode in Kelvin and $q=1.6 \times 10^{-19} C$ is the charge represented by an electron. Finally, $R_{s}$ is the equivalent series resistance of the PV array describing an internal resistance to the current flow.

All the parameters can be determined by equation (3) with the subscript $r$ represents reference. $E$ is irradiation, $K_{0}$ is the temperature coefficient of the short circuit current which can be found from the manufacture's data sheet together with $T_{r}, E_{r}$ and short circuit current $I_{s c r}$, $V_{g}$ is the band gap voltage of the semiconductor, finally, term $\frac{d V}{d I_{V_{O C}}}$ can also be generated from manufacturer's data sheet.

$$
\left.\begin{array}{l}
I_{p}=I_{p r}+K_{0}\left(T-T_{r}\right) \\
I_{p r}=I_{s c r} \frac{E}{E_{r}} \\
I_{s}=I_{s r}\left(\frac{T}{T_{r}}\right)^{\frac{3}{\varepsilon}} \exp \left(-\frac{q V_{g}}{\varepsilon k_{B}}\left(\frac{1}{T}-\frac{1}{T_{r}}\right)\right) \\
I_{s r}=\frac{\exp \left(q V_{O C r} / \varepsilon k_{B} T_{r}\right)-1}{d V}-\frac{1}{d I_{V_{O C}}} X_{V} \\
R_{s}=-\frac{q}{\varepsilon k_{B} T_{r}} \exp \left(\frac{q V_{O C r}}{\varepsilon k_{B} T_{r}}\right) \\
\left.X_{V}=I_{s r} \frac{1}{d V}\right)
\end{array}\right\}
$$

The seconder energy source is the battery pack which creates a DC connection between all components in the solar powered self-sustainable energy system. It is used as an energy store and buffers fluctuations in storage and compensates for fluctuation in power generation by the PV cells. The battery state of charge $S$ is the only state variable within the battery system model and is defined as;

$$
S=\frac{Q_{\text {max }}-\int_{0}^{t} I_{b} d t}{Q_{\max }}
$$

where $Q_{\text {max }}$ is the battery's maximum capacity. The battery current $I_{b}$ is defined by;

$$
I_{b}=-\left(I_{P V}-I_{\text {Load }}\right)
$$

where $I_{P V}$ is the PV array's current and $I_{\text {Load }}$ is the load current. When charging the battery the current is positive, and conversely a negative current indicates that the battery is discharging. It is typically found that the length of a battery's life maybe extended by avoiding overcharging and deep discharging. Knowing the $Q_{\max }$ is important to determine the size of the battery pack, which can support the load demand of a given period.

The key role of the energy management system is to make full use of the power generated from solar irradiation and minimising the use of stored energy in the battery pack, i.e., to stop the batteries state of charge (SoC) falling below its midpoint, to keep the battery state of charge (SoC) at around midpoint, which will extend its lifespan and increase the overall system efficiency. Therefore, with carefully selected controller design system, all the power generated by the solar PV array will be used either to support the load demand or to charge the battery pack, alternatively feedback to the sustainable system environment, which maximise the usage and minimise the operating cost. 


\section{Intelligent Controller}

In this section, the design of intelligent controllers for sustainable energy systems is described, in particular for home energy system based on PV-BESS (PV- Battery Energy Storage System) components. In this intelligent controller design, fuzzy logic mechanism is selected to address the energy management issues.

There are three major control subsystem loops in the system that regulates the energy demand / supply, the energy management and the energy storage. Several researchers have addressed EMS and the design and applications of renewable energy systems [2]. In this study, the target of the control strategy is to use the solar energy to meet the energy demand, then utilise any additional energy to maximise the storage and minimise the usage of battery at the same time. Therefore, the overall system's efficiency will be increased and lifetime of the battery pack will be extended. The operating strategies for energy management system are as follows; firstly, on the basis of the efficiency, the highest priority is to utilise solar power to satisfy the energy demand. If excess solar energy is available, it will be sent to BEES, which will be compressed and stored for future use in electrical energy generation. If there is still additional energy generated by the PV array, when the load demand is satisfied, the extra solar energy will be utilised to charge the battery, if the battery SoC is sufficiently low. If the energy generated by the PV array is insufficient to support the electrical energy demand, the different is to be supplied by the BESS, hence provide a short-term compensation. It should be noted that with carefully selected system components, all the energy generated by solar PV array will be used either to support the load demand, or to charge the battery pack. The required control objects are governed by two-input-one-output fuzzy logic controller. The two inputs to the fuzzy logic controller are the differential energy flow $(\mathrm{dE})$ and the battery's SoC. The differential energy flow represents the difference between available solar energy and the energy demand. The controller will use this information to decide whether excess solar energy is available for storage. The SoC input is used to maintain the battery's $\mathrm{SoC}$ at a desired level in order to prevent overcharging or overly deep discharge of the battery pack. The fuzzy mechanism consists of triangular membership functions for the two inputs and for the one output. The reason for choosing a triangular membership function is simplicity and ease of computation. The $\mathrm{dE}$ flow is divided into eight variables. A negative energy supply means that the battery is required to supply the difference and positive energy means additional solar energy can be used to charge the battery. The battery SoC is also described by three variables. It is desirable that the $\mathrm{SoC}$ be maintained within a region of between $50 \sim 60 \%$. The rules of the fuzzy logic controller are presented in Table 1 and the definitions of the linguistic variables are elaborated in Table 2.

\begin{tabular}{|l|l|l|l|l|l|l|l|l|l|}
\hline \multicolumn{2}{|c|}{} & NL & NM & NS & $Z$ & PS & PM & PL & PEL \\
\hline \multirow{3}{*}{ SOC } & L & NL & NL & NM & NS & Z & PS & PM & PL \\
\cline { 2 - 9 } & C & NL & NM & NS & Z & PS & PM & PL & PEL \\
\cline { 2 - 9 } & H & NM & NS & Z & PS & PM & PL & PEL & PEL \\
\hline
\end{tabular}

Table 1: Basic rules table of the fuzzy inference.

In Table 1, the top row of the table shows the differential of energy flow (dE) and the left column is the battery's SoC. The cells of the table at the intersection of rows and columns contain the linguistic value for the output corresponding to the first input written at the beginning of the row and to the value of the second input written on the top of the column. The rule output was defuzzified using a centroid computation.

Table 2: Linguistic variables in the fuzzy inference system

\begin{tabular}{|l|l|}
\hline Linguistic & Linguistic meanings \\
\hline NL & Negative Large \\
\hline NM & Negative Medium \\
\hline NS & Negative Small \\
\hline Z & Zero \\
\hline PS & Positive Small \\
\hline PM & Positive Medium \\
\hline PL & Positive Large \\
\hline PEL & Positive extreme Large \\
\hline L & Low \\
\hline C & Correct \\
\hline H & High \\
\hline
\end{tabular}

\section{Results and Discussion}

The results based on real data indicates that the proposed controller can direct the energy flow wisely between different elements such as load and battery, it is capable of meeting the daily energy demand and using the excess solar energy to store it while maintaining the battery's health and stage of charge in a desired region in order to extend the lifespan of the BESS.

The annual solar power delivered by the PV system and the demand are shown in Figure 4 and 5. It can be seen from the figures, that the fluctuation of power can be 0.1 $-18 \mathrm{~kW}$. Therefore, a suitable energy storage system is key for successful implementation of self-sustainable energy system. In this work a battery pack is selected as an energy storage system and the initial $\mathrm{SoC}$ was set at $50 \%$. Form the Figure 6, it can be seen that the intelligent controller can maintain the battery SoC around 55\% during the year as desired, which help to increases the efficiency of the whole system. 


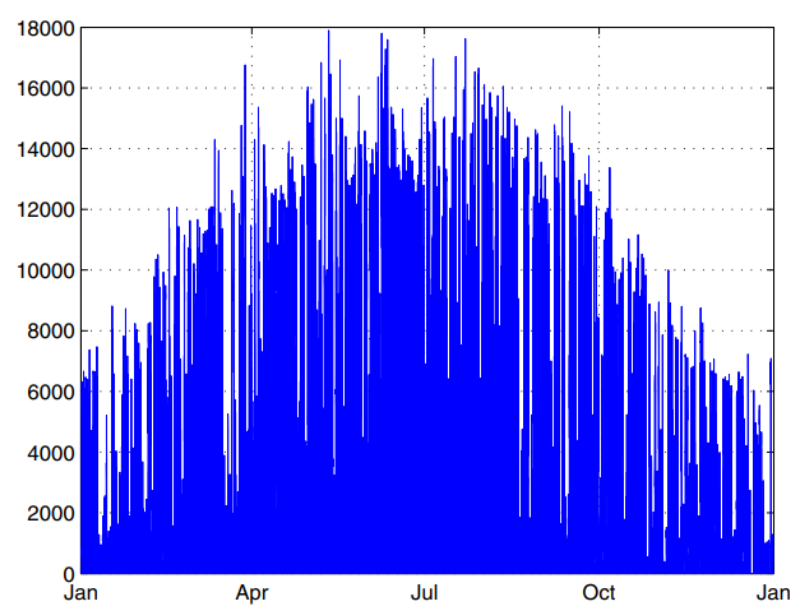

Fig.4. Solar energy generation (year)

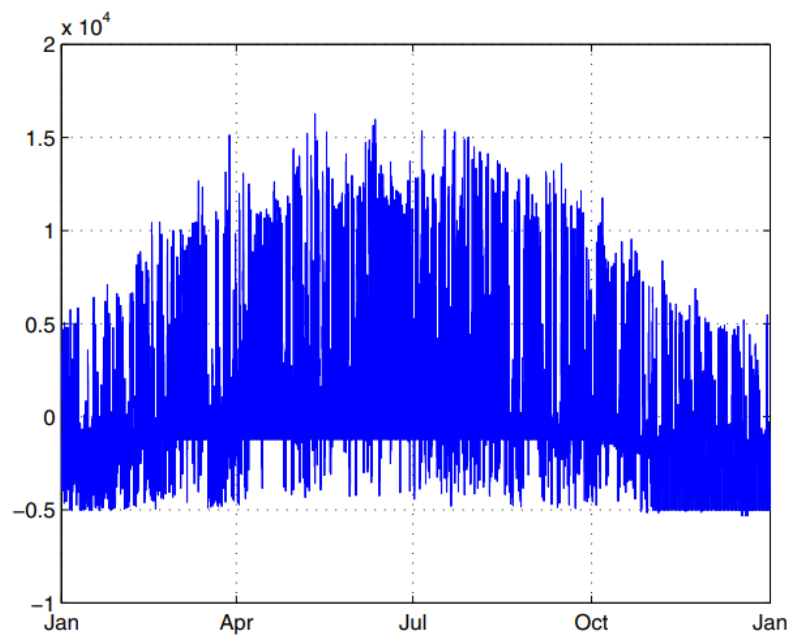

Fig.5. Different of supply/demand (year)

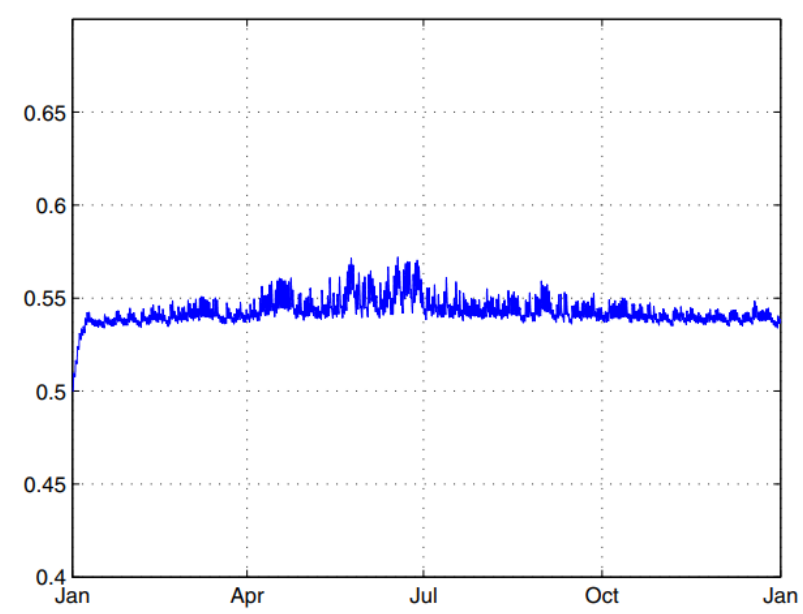

Fig.6. Battery - State of Charge (year)

\section{Concluding remarks}

In this paper, solar powered self-sustainable energy system with efficient energy usage is investigated. For such system energy management is optimised with the use of an intelligent controller. This work also described an optimal design and implementation of an efficient selfsustainable energy system with consideration of energy storage options for excess energy produced by renewable energy sources. The analysis presented in this paper is indicated that for small-scale system battery energy storage is a best option.

\section{References}

[1] E. Constant, K. Thanapalan, M. Bowkett. Optimal energy storage evaluation of a solar powered sustainable energy system. SICEISCS 2018 IEEE International Conference, 193 - 198, 2018.

[2] A. I. Dounis, C. Caraiscos. Advanced control systems engineering for energy and comfort management in a building environmnet A review. Renewable and Sustainable Energy Reviews, vol.13, issue 6-7, pp.1246-1261, 2009.

[3] K. Thanapalan, F. Zhang, S. Carr, G. Premier, A. Guwy, J. Maddy, 'An overview of renewable energy technologies and hydrogen economy' Renewable Energy and Power Quality Journal, vol. 1, no. 11, pp. 322:1-6, March. 2013

[4] M. A. S. Masoum, H. Dehbonei, E. F. Fuchs, 'Theoretical and experimental analyses of photovoltaic systems with voltage and current-based maximum power-point tracking', IEEE Transactions on Energy Conversion, vol. 17, no. 4, pp. 514-522, 2002.

[5] V. Salas, E. Olías, A. Barrado, A. Lázaro, 'Review of the maximum power point tracking algorithms for stand-alone photovoltaic systems', Solar Energy Materials and Solar Cells, vol. 90, no. 11, pp. 1555-1578, Jul. 2006. Energy Storage in photovoltaic stand-alone energy

[6] G. Bopp, H. Gabler, K. Preiser, D. U. Sauer, H. Schmidt, 'Energy storage in photovoltaic stand-alone energy supply systems', Progress in Photovoltaics: Research and Applications, vol. 6, no. 4, pp. 271-291, 1998.

[7] Evans A, Strezov V, Evans TJ (2012) Assessment of utility energy storage options for increased renewable energy penetration. Renew Sustain Energy Rev 16:4141-4147.

[8] Yekini Suberu M, Wazir Mustafa M, Bashir N (2014) Energy storage systems for renewable energy power sector integration and mitigation of intermittency. Renew Sustain Energy Rev 35:499-514

[9] A. Gonzalez, E. Goikolea, J.A. Barrena, R. Mysyk. Review on supercapacitors: technologies and materials. Renewable and Sustainable Energy Reviews, vol.58, pp.1189-1206, 2016.

[10] P.F. Ribeiro et al.,. Energy Storage Systems for Advanced Power Applications. "Proceedings of the IEEE," vol.89, issue 12, pp. 1744-1746, 2001

[11] X. Li, D. Hui, M. Xu, L. Wang, G. Guo, L. Zhang. Integration and energy management of large-scale lithium-ion battery energy storage station. 2012 15th international conference on electrical machines and systems (ICEMS), Sapporo, Japan., pp. 1-6, 2012.

[12] LATimes. Tesla builds world's largest batter in Australian outback. Technology, Associated Press, Dec 2017.

[13] I. Hadjipaschalis, A. Poullikkas, V. Efthimiou. Overview of current and future energy storage technologies for electric power applications. Renewable and Sustainable Energy Reviews, 13(67), pp.1513-1522, 2009

[14] K. Appunn. Sector coupling - shaping an integrated renewable energy system. Journalism for the Energy Transition. Clean Energy Wire CLEW, 2018.

[15] F. Zhang, S. Carr, K. Thanapalan, J. Maddy, A. Guwy. Design and Simulation of A Single Current Sensor Maximum Power Point Tracker for Solar Hydrogen System. Renewable Energy and Power Quality Journal, Vol.11, no. 333, 2013. 